INNOVATION

\title{
L'IGR lance sa première RCP moléculaire
}

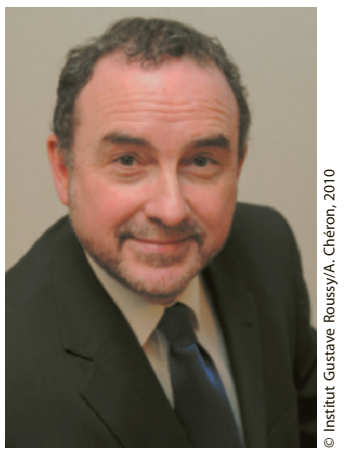

Pr Gilles Vassal

Directeur de la recherche clinique et translationnelle en charge du projet médical Institut Gustave Roussy

114, rue Edouard-Vaillant - 94805 Villejuif

\section{Quel est le contexte actuel de la méde-} cine personnalisée en cancérologie ?

Dans certaines pathologies, et ce depuis plusieurs années déjà, les données biologiques concernant la nature des tumeurs sont prises en compte pour adapter certains traitements. C'est par exemple le cas pour les neuroblastomes, des tumeurs rares chez l'enfant, certaines leucémies et bien sûr le cancer $d u$ sein, pour lequel on recherche la présence ou non du récepteur Her2. De nos jours, de plus en plus de médicaments innovants nécessitent, pour être utilisés à bon escient, que l'on identifie les patients qui peuvent en bénéficier, c'est-à-dire ceux dont la tumeur va répondre au traitement. C'est ainsi que I'INCa a tout récemment mis en place un programme de soutien des 28 plateformes hospitalières de génétique moléculaire pour qu'elles détectent dès à présent en routine un panel de biomarqueurs : il s'agit de rechercher, pour le cancer colorectal, les patients dont la tumeur présente une forme non mutée du gène KRAS, et pour le cancer du poumon, les patients dont la tumeur présente une mutation activatrice $d u$ gène de l'EGFR. Cette pratique du diagnostic moléculaire est appelée à se développer, et pas seulement pour les nouveaux médicaments, cela concerne aussi la chimiothérapie, la radiothérapie et même la chirurgie.

\author{
Quels sont les enjeux de cette médecine \\ dite " personnalisée » ?
}

Autrefois, la caractérisation des tumeurs se faisait essentiellement d'un point de vue anatomopathologique. Désormais, il s'agit aussi d'identifier des biomarqueurs moléculaires et génétiques qui nous permettent de réserver les traitements aux patients chez qui ils vont avoir une certaine efficacité, et d'éviter ainsi aux autres patients des effets secondaires inutiles mais aussi des dépenses superflues. Cela concerne toute l'oncologie. Grâce au formidable essor des technologies à haut débit comme la génomique qui génère des millions d'informations, on peut guider la prise en charge des patients de façon plus précise et surtout plus pertinente. C'est un véritable changement en profondeur pour l'oncologie, et pas seulement dans le domaine des médicaments innovants.

Est-ce réaliste scientifiquement et économiquement de vouloir proposer ça à tous les patients?

À court terme, non car il faut d'abord en démontrer la faisabilité, valider le principe dans des études de recherche clinique. À moyen terme, on devrait pouvoir proposer cela à une grande majorité de patients, concernés par les pathologies les plus fréquentes mais aussi pour des cancers rares. Car chaque jour apporte son lot de nouvelles connaissances sur la biologie des tumeurs et de nouvelles thérapeutiques. Par ailleurs, il faut bien prendre conscience que cette approche permet en quelque sorte une rationalisation des thérapeutiques mises en œuvre. Certes tous les nouveaux médicaments coûtent chers, et certains même très chers, mais s'ils ne sont utilisés que chez les patients dont la tumeur va répondre, alors il n'y a rien à dire ! Il faut une approche raisonnée et raisonnable de cette médecine personnalisée. Pour cela, les outils nécessaires à ces diagnostics moléculaires, en l'occurrence les plateformes à haut débit, doivent évidemment se développer au même rythme que les innovations thérapeutiques sinon c'est illusoire de vouloir proposer ces nouveaux médicaments au plus grand nombre.

\section{Quels sont les objectifs de I'IGR en la matière? \\ Dans un premier temps, il s'agit de faire} la preuve du concept d'une thérapie guidée par la biologie et par l'image et d'offrir une prise en charge personnalisée à un patient devenu partenaire. Mais nous souhaitons aussi évaluer l'impact psychosocial et médicoéconomique de cette approche et d'être actif dans la recherche en participant au développement de nouveaux médicaments et leur biomarqueurs grâce à notre Service d'Innovation Thérapeutique Précoce (SITEP). Dans le cadre du développement de cette médecine personnalisée, l'approche pangénomique de la tumeur nous paraît la plus pertinente. C'est-à-dire qu'au lieu de multiplier les tests sur des marqueurs moléculaires un par un, nous souhaitons dès le départ dresser un portrait moléculaire de la tumeur pour avoir le maximum d'informations.

\section{Concrètement, comment allez-vous procéder?}

Nous avons d'ores et déjà mis en place des réunions de concertation pluridisciplinaires $(R C P)$ moléculaires où le meilleur traitement pour un patient est discuté en prenant compte aussi de son portrait moléculaire tumoral. C'est une expérience pilote qui se déploie actuellement en pneumologie et en pathologie mammaire pour les formes avancées métastatiques. L'enjeu est majeur puisqu'il concerne aussi bien les moyens financiers qu'humains dont nous disposons! II nous faut repenser les plateformes biologiques, trouver de nouvelles ressources, mettre en place de nouveaux circuits. Pour 2010, nous espérons traiter 400 dossiers de nouveaux patients lors de $R C P$ moléculaires, puis monter en puissance les années suivantes. Notre objectif est de valider la pertinence scientifique et économique de cette approche et de la déployer.

Propos recueillis par Émilie Gillet 